\title{
Evaluation of High-Resolution STEM Imaging Advancement Under Gas- Environment with Open Window MEMS Holder and Gas Injection System
}

\author{
Akinari Hanawa ${ }^{1 *}$, Yudai Kubo ${ }^{1}$, Hideki Kikuchi ${ }^{1}$, Kuniyasu Nakamura ${ }^{1}$, Manabu Shirai ${ }^{1}$, Hiromi \\ Inada $^{1}$, Hiroaki Matsumoto ${ }^{2}$ and Masahiro Kawasaki ${ }^{3}$ \\ 1. Science System Product Division, Hitachi High-Technologies Corporation, Hitachinaka, Ibaraki, Japan. \\ 2. Hitachi High-Technologies Co., Ltd., Chao Yang District, Beijing, China. \\ ${ }^{3 .}$ Hitachi High-Technologies America Inc., Clarksburg MD, USA. \\ * Corresponding author: akinari.hanawa.gv@hitachi-hightech.com
}

Observation of reaction processes in the in-situ environment has been increasingly demanded to understand the properties of catalysts and fuel cells with a transmission electron microscope (TEM). Insitu TEM / STEM play an important role to analyze the structural changes at the atomic scale under the heating and gas environments. Furthermore, with the size shrinkage of the target materials or devices, in-situ real time imaging with a sub-Angstrom resolution has become more demanded. The analytical $200 \mathrm{kV}$ cold field emission (CFE) TEM HF5000 (Hitachi High-Technologies) equipped with an inhouse designed probe-forming aberration corrector was introduced and the imaging performance of the environmental real time scanning transmission electron microscopy (STEM) was reported [1][2]. The HF5000 key feature of in-situ STEM is a live scanning acquisition (25 frames/sec) that allows real-time observation and video recording of dynamic atomic scale reactions. HF5000 provides clear real time imaging for such as structure change and elemental diffusion processes during reaction. Besides DF / BF live images, a secondary electron image in the atomic scale can be simultaneously acquired with the rich high resolution surface morphology information in-situ.

The HF5000 can have two gas injection nozzles, one is attached in the specimen chamber and the other is in a filament-heating holder. The gas can be chosen independently (Figure 1). Gas flow is limited from one nozzle at a time and can be controlled from the HF5000 GUI. Maximum allowable flow rate is $3.5 \mathrm{sccm}$. The calibrated local pressure around the specimen is $10 \mathrm{~Pa}$ at the maximum flow. Compared to the conventional environmental cell holder system, the gas flow at the sample area is flexible and reproducibility of the flow rate is high because the environmental cell that the HF5000 system provides is confined by the differential aperture. Also, there is no risk of membrane breakage.

Figure 2 shows the electron beam scattering rate as a function of the pressure. The electron beam scattering rate that $200 \mathrm{kV}$ electrons pass through the air layer of $5 \mathrm{~mm}$ distance was calculated by Monte Carlo simulation. Scattering rate is defined as the ratio of the number of unscattered electrons to the number of incident electrons. The vertical axis is normalized as the scattering rate at $5 \times 10^{-5} \mathrm{~Pa}$ is indicated as 1 . At a pressure higher than $10 \mathrm{~Pa}$, electron beam scattering events occur, and at $5000 \mathrm{~Pa}$ about $50 \%$ of electron beam is scattered. In case of the HF5000, the vacuum pressure in the vicinity of the specimen should be limited to $10 \mathrm{~Pa}$ at maximum, and therefore it is assumed and proved that atomic resolution imaging without being affected by electron beam scattering is possible.

In addition, the chip-based sample holder is developed using microelectromechanical systems (MEMS) technology (Figure 3), by Norcada and Hitachi High-Technologies Canada. Maximum heating temperature of this holder is 1100 degree C. Minimum controllable temperature increment is 1 degree C. Heating up speed from the room temperature to 1000 degree is as fast as a few seconds. MEMS heater 
has been proved to minimize the specimen drift because the heated area is so small to reduce the thermal expansion quickly. There are thin areas on a SiNx thin membrane film. They are the holes of approximately 3 micrometer in diameter and used to drop the specimens on for high resolution STEM observation. In combination with the gas injection nozzle provided in the HF5000 specimen chamber and a MEMS holder, atomic resolution in-situ observation under the high gas pressure and heating environment is safely enabled.

Figure 4 shows an example of high resolution STEM under the gas pressure below $3 \mathrm{~Pa}$ and a temperature of 200 degree $\mathrm{C}$ provided by the specimen chamber gas injection system and the MEMS heating holder. It is demonstrated that the information for the atomic assignment of a catalytic particle $\mathrm{Pt}$ on a supporting $\mathrm{CeO}_{2}$ mother particle is clearly obtained by HAADF STEM and SEM. This combined technique of in situ STEM/SEM and a MEMS heating holder provides the high resolution surface morphology of catalyst.

References:

[1] H Inada et al., Microsc. Microanal. 24 (2018), p. 318.

[2] H Inada et al., Microsc. Microanal. 23 (2017), 918.

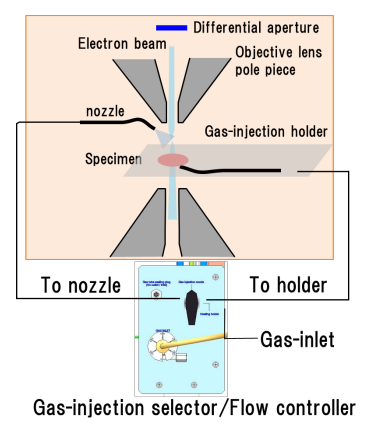

Figure 1. A schematic diagram of Figure 2. Electron beam scattering rate as a function of Gas injection system suitable for environmental (S)TEM imaging.

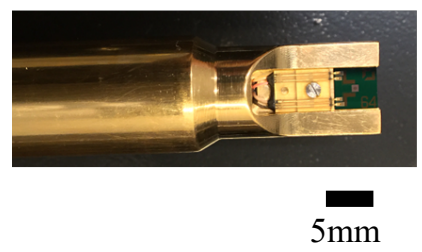

pressure.
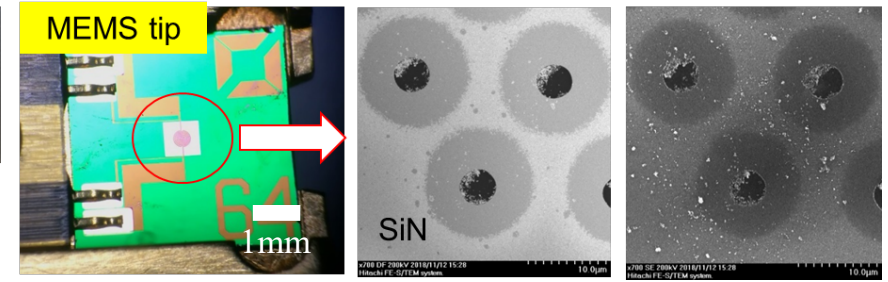

Figure 3. Outline drawing of MEMS heating holder
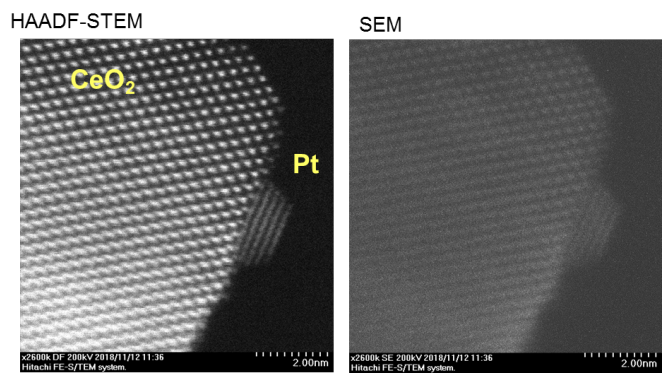

Figure 4. High-resolution HAADF-STEM and SEM observation using a MEMS heating holder and an in-chamber nozzle. Sample $\mathrm{Pt} / \mathrm{CeO}_{2} / \mathrm{C}$ catalysts at a gas pressure up to $3 \mathrm{~Pa}$ and a temperature of 200 degree C 